\title{
THE NORTHROP GRUMMAN COMPACT INFRARED FEL (CIRFEL)*
}

\author{
I. S. Lehrman, J. Krishnaswamy, R. A. Hartley \\ Northrop Grumman Advanced Technology and Development Center, 4 Independence Way, Princeton, \\ New Jersey 08540, USA \\ R. H. Austin \\ Physics Department, Princeton University, Princeton, New Jersey 08544, USA
}

\section{INTRODUCTION}

As part of Northrop Grumman's ongoing research in particle accelerators, we have designed and are commissiong a Compact Infrared Free-Electron Laser (CIRFEL) for the study of high-brightness electron beams and free-electron lasers. Besides serving as a tool for FEL development, the CIRFEL laboratory, located at Princeton University, will be used in experiments ranging from basic FEL physics and biophysics to chemistry, materials science and medicine.

The CIRFEL is to lase initially in the $10-20 \mu \mathrm{m}$ range. The pulse format of this FEL is a train of micropulses, $5-10 \mathrm{psec}$ is duration, at a repetition rate of $142.8 \mathrm{MHz}$. The micropulse energy is in excess of $100 \mu \mathrm{J}$. The micropulses comprise a macropulse lasting approximately $10 \mu \mathrm{sec}$. The macropulse repetition rate is $10 \mathrm{~Hz}$, thus the average power of the FEL is on the order of $1.5 \mathrm{~W}$.

\section{SYSTEM DESCRIPTION}

The CIRFEL consists of the following system components: (1) a high-brightness photocathode electron gun, (2) a photocathode illumination laser, (3) a $30 \mathrm{MW}$ S-band RF source, (4) the beam transport system, (5) the FEL wiggler, (6) the FEL optical cavity, and (7) the associated support hardware subsystems. Figure 1. shows the layout of the CIRFEL beamline.

To allow for flexibility and possible future expansion, the beamline components are mounted on a pair of rails. The rails allow the axial positions of the beamline elements to be easily adjusted. The rails are mounted to nonmagnetic optical tables which allows additional optics and diagnostics to be fixed to the tables with standard mounts. A description of each of the system components follows.

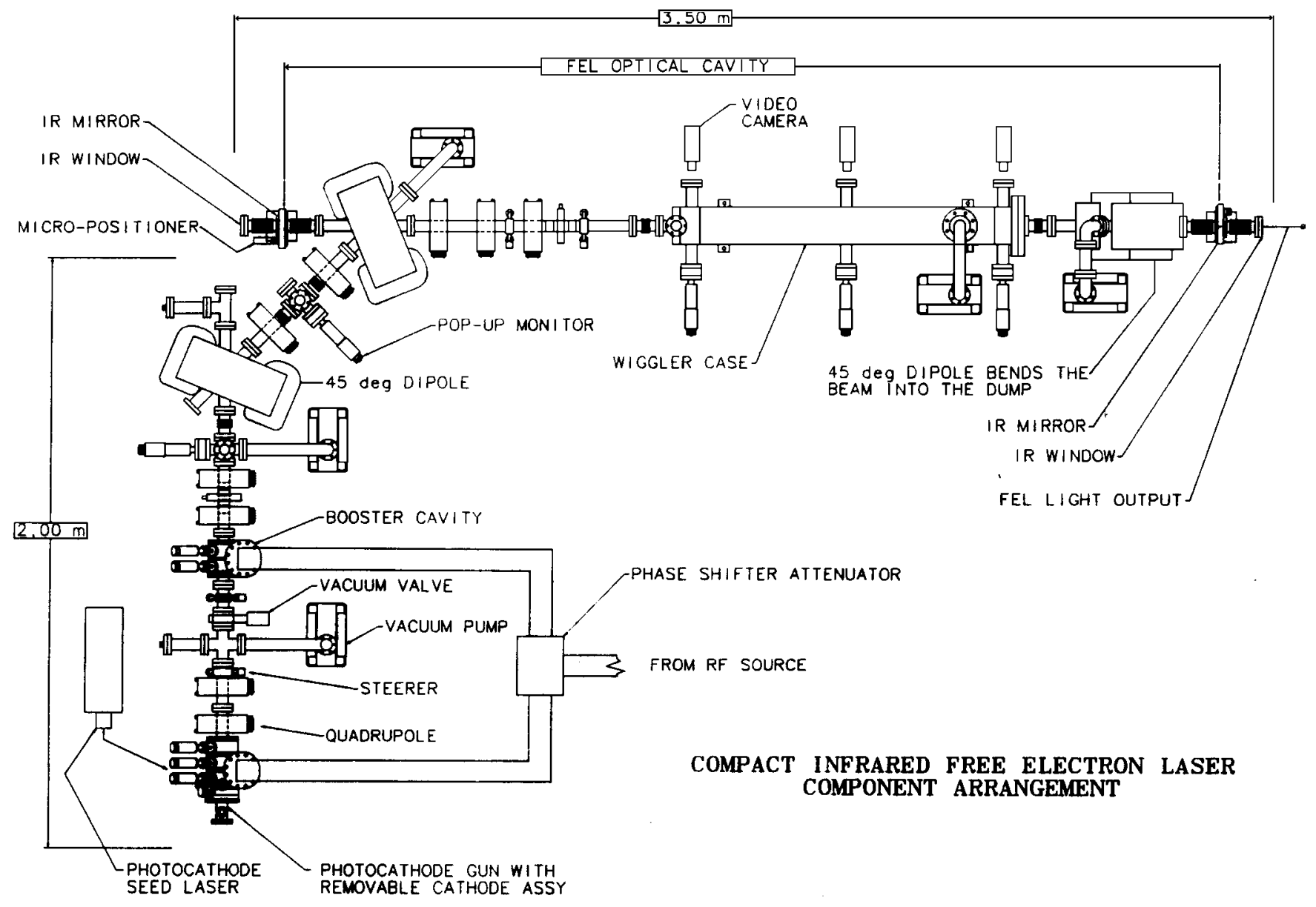

Figure 1. Layout of the CIRFEL beamline. 


\section{A. High-Brightness Photocathode Electron Gun}

The accelerator for the CIRFEL is a photocathode electron gun shown in Figure 2. The gun is only $20 \mathrm{~cm}$ long and produces electron beams with energies up to $9 \mathrm{MeV}$. The gun consists of $3^{1 / 2}$ cells operated in the $\pi$-mode phasing. The gun is fed from a single waveguide which couples power directly to cells 2 and 3 . Power is aperture coupled from cell 2 to cell 1 and from cell 3 to cell 4 .

The gun has been modeled extensively with the MAGIC[1] particle-in-cell code. These results indicate that the beam emittance (RMS) will be between 2.7 and $10 \pi \mathrm{mm}$ mrad depending upon the launch phase. The peak electric field at the cathode will be between $60-80 \mathrm{MV} / \mathrm{m}$ with gradients up to $100 \mathrm{MV} / \mathrm{m}$ in cells 2 through 4 .

The photocathode, which is contained in the first cell, is a $6 \mathrm{~mm}$ diameter disc of Mg. Approximately $7 \mu \mathrm{J}$ of $\mathrm{UV}$ $(262 \mathrm{~nm})$ is required to release $1 \mathrm{nC}$ of charge.[2]

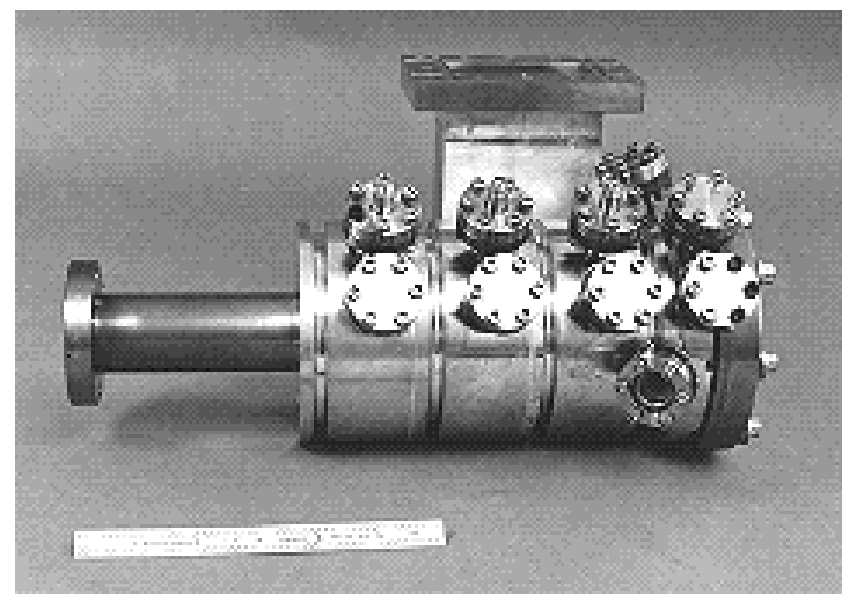

Figure 2. CIRFEL Photocathode Electron Gun

Downstream of the photocathode gun, we have installed a two cell booster cavity to increase the beam energy to $14 \mathrm{MeV}$. This cavity is identical to the central two cells of the gun.

\section{B. Photocathode Illumination Laser}

The photocathode laser for the CIRFEL gun consists of a ND:YLF mode-locked oscillator, a pulse slicer, two double-passed stages of amplification, and harmonic generation. The mode-locked oscillator is a Lightwave Electronics' diode-pumped unit operating at $142.8 \mathrm{MHz}$. The oscillator produces $7 \mathrm{psec}$ IR pulses $(1047 \mathrm{~nm})$ at an average energy of $200 \mathrm{~mW}$. The temporal jitter of the oscillator has been measured to be less than 1 psec.

The oscillator was integrated into a macropulse amplifier by Continuum. A pulse slicer is used to slice out a $10 \mu \mathrm{sec}$ portion of the beam. The beam is then double-passed through a $7 \mathrm{~mm}$ flash lamp-pumped ND:YLF amplifier. After spatially filtering the beam, the beam is passed through another $7 \mathrm{~mm}$ ND:YLF amplifier. Second and fourth harmonic generation crystals are used to produce $524 \mathrm{~nm}$ and $262 \mathrm{~nm}$ radiation. The laser is capable of producing in excess of $16 \mu \mathrm{J}$ per micropulse at $262 \mathrm{~nm}$.

\section{S-Band RF Source}

The RF system consists of a $700 \mathrm{~W}$ S-band amplifier (IPA) which feeds a 30 MW ITT 2960 klystron. The modulator for the klystron has a 13 stage pulse forming network (PFN) which produces a video pulse of $12 \mu \mathrm{sec}$ (FWHM). The klystron has a flattop output of $10 \mu \mathrm{sec}$. The RF power from the klystron is split by a power divider to feed both the gun and booster. In order to correct for the PFN ripple, we have implemented Feed-Forward ${ }^{3}$ correction for both the RF amplitude and phase. The RF amplitude and phase are sampled in cell 1 of the gun. Amplitude and phase corrections are programmed into arbitrary waveform generators (AWG). The AWG's drive a pin-diode antenuator and phase shifter which are in the low-level RF chain driving the IPA.

\section{Beam Transport System}

The TRACE3D[4] and PARMELA[5] beam optics/dynamics codes were used to model the CIRFEL beamline. A quadrupole doublet is used to focus the beam from the output of the gun. An achromatic bend is used to allow for mirror access for the FEL optical cavity. Depending upon the longitudinal phase space distribution of the beam, the achromat can act to compress the longitudinal length of the beam, thus increasing the peak current of the bunch.

A quadrupole triplet is used to focus the beam into the wiggler. In order to reduce horizontal defocusing effect of the $45^{\circ}$ bending magnets, the poles were cut with $22.5^{\circ}$ entrance angles. Once a TRACE3D solution was found for the beamline, the PARMELA code was used to determine the effect of space charge. PARMELA simulations indicate there is no significant space charge induced emittance growth for 1 $\mathrm{nC}$ bunches. Space charge will cause some slight changes in the effective focal lengths of some of the quadrupoles.

\section{E. FEL Wiggler}

Initial experiments will be carried out with a $1.36 \mathrm{~cm}$ period, 73 period permanent magnet wiggler loaned from the Los Alamos National Laboratory. The wiggler gap is $7 \mathrm{~mm}$ and the RMS wiggler parameter, $A_{W}=0.16$. Our calculations indicated that the single pass gain should be in excess of $50 \%[6]$.

\section{F. FEL Optical Cavity}

The optical cavity is a near concentric, asymmetric stable cavity, with a Rayleigh range of $\sim 0.5 \mathrm{~m}$. The cavity utilizes copper mirrors with central holes for outcoupling the radiation. A HeNe alignment laser, which is injected in the upstream mirror, is used to align the electron beam onto monitors that are inserted into the wiggler. The HeNe laser light which is outcoupled through the downstream mirror is used to align the cavity mirrors. $\mathrm{ZnSe}$ output windows are used at the vacuum interface.

\section{STATUS}

Figure 3. shows the CIRFEL device in its present configuration. As of April 1995, we have installed all of the beamline including the wiggler. The cavity length was determined by injecting the ND:YLF mode-locked laser into the cavity and observing interference between sucessive pulses. 


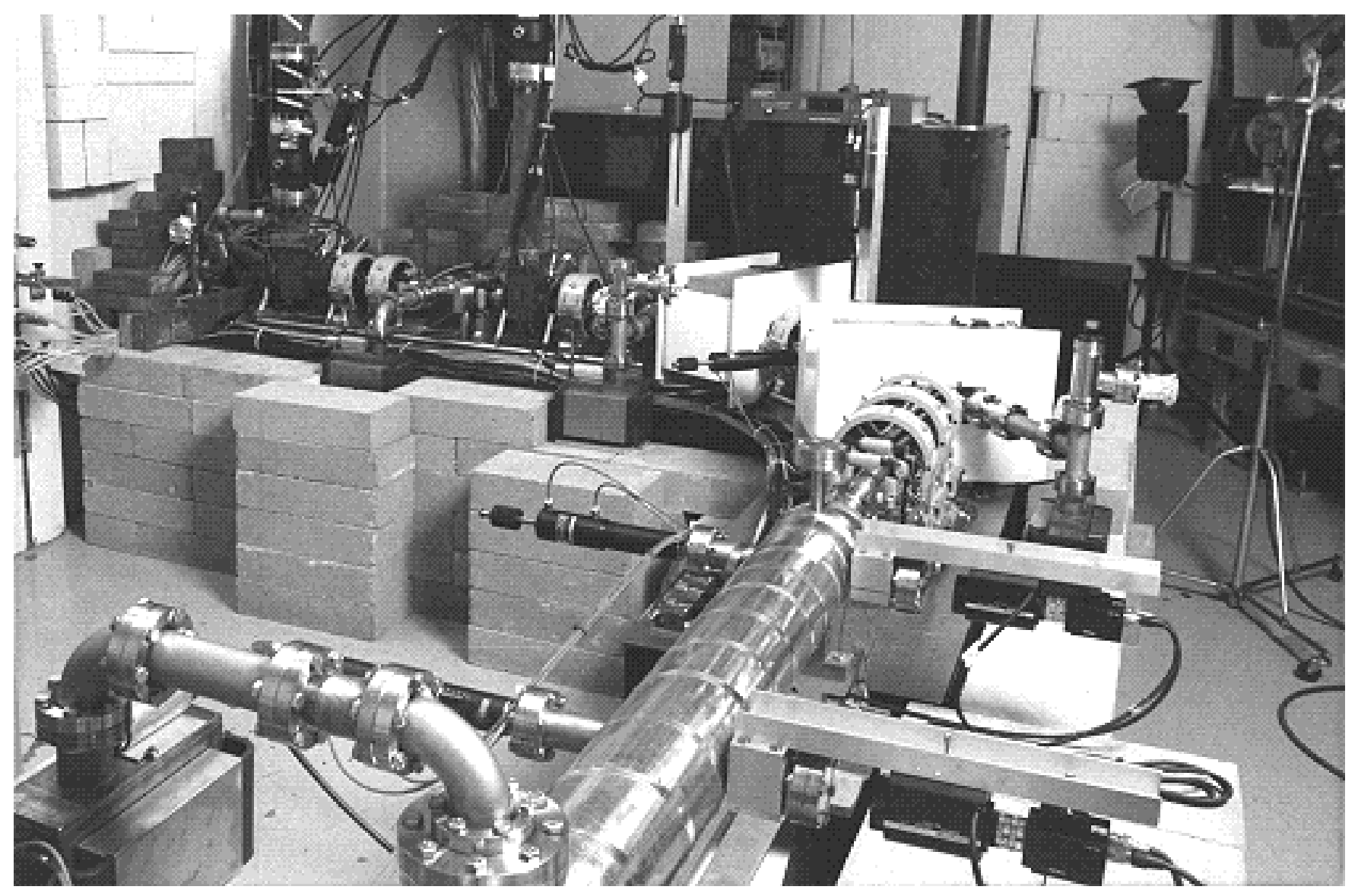

Figure 3. CIRFEL Device installed at Princeton University. The gun is located in the top left corner and the wiggler is contained in the vacuum case in the center of the figure.

From the amplitude of the interference signal, we were able to determine the cavity length to within 50 microns. Using the RF Feed-Forward correction scheme, we have flattened the RF amplitude in the gun to better than $0.1 \%$ and the RF phase to 1 degree. In excess of $1 \mathrm{nC}$ of charge at 11 $\mathrm{MeV}$ has been transported through the wiggler with $100 \%$ transmission. Spontaneous emission has been routinely observed, but we did not see any signs of optical gain.

Beam position measurements indicated that the wiggler axis was not properly aligned to the electron beam and optical cavity axis. An examination of the wiggler revealed a bow in the wiggler structure of $0.75 \mathrm{~mm}$ at the center. The wiggler has been removed and will be reassembled and measured at the Los Alamos National Laboratory. At re-assembly, the wiggler gap will be reduced to $6 \mathrm{~mm}$ in order increase the gain from the predicted value of $50 \%$ to nearly $100 \%$ (including degradation effects due to emittance and energy spread).

Once the wiggler is re-installed, we will be working to observe optical gain and then demonstrate FEL lasing.

\section{REFERENCES}

[1]G. D. Warren, L. Ludeking, J. McDonald, K. Nguyen, and B. Goplen, "MAGIC Users Group Software," Proceedings of the Conference on Computer Codes and the Linear
Accelerator Community, Los Alamos National Laboratory, Jan 22-25, 1990, Richard Cooper, Ed. LA-11857-Z (1990) 57.

[2]P. G. O'Shea, to be published in the 1994 Proceeding of the International Free-Electron Laser Conference, Nucl Inst and Meth A. (1995)

[3] R. Zhang, I. Ben-Zvi and J. Xie, Nucl Inst. Meth A324 (193) 421-428.

[4] K. R. Crandall, Los Alamos National Laboratory report LA-11054-MS, August 1987.

[5] K. R. Crandall and L. Young, "PARMELA," in the Compendium of Computer Codes for Particle Accelerator Design and Analysis, H. Deaven and K. C. Chan, Eds. Los Alamos Natl. Lab report LA-UR-90-1766, May (1990) 137.

[6] "FEL CAD," D. C. Nguyen, S. Gierman and P. O'Shea, available from Los Alamos National Laboratory, unpublished 1994.

*This work is supported by the Northrop Grumman Advanced Technology and Development Center 